\title{
Grounding at a distance
}

\author{
Sam Baron ${ }^{1} \cdot$ Kristie Miller $^{2} \cdot$ Jonathan Tallant $^{3}(\mathbb{D}$
}

\begin{abstract}
What distinguishes causation from grounding? One suggestion is that causation, but not grounding, occurs over time. Recently, however, counterexamples to this simple temporal criterion have been offered. In this paper, we situate the temporal criterion within a broader framework that focuses on two aspects: locational overlapping in space and time and the presence of intermediaries in space and time. We consider, and reject, the idea that the difference between grounding and causation is that grounding can occur without intermediaries. We go on to use the fact that grounding and causation both involve intermediaries to develop a better temporal criterion for distinguishing causation from grounding. The criterion is this: when a cause and effect are spatially disjoint, there is always a chain of causal intermediaries between the cause and the effect that are extended in time. By contrast, when the grounds and the grounded are spatially disjoint, there is always a chain of grounding intermediaries, but the chain need not be extended in time, it can be purely spatial. The difference between grounding and causation, then, is that causation requires time for chaining in a way that grounding does not.
\end{abstract}

Keywords Grounding · Causation · Dependence · Location · Time

Jonathan Tallant

jonathan.tallant@nottingham.ac.uk

Sam Baron

samuel.baron@uwa.edu.au

Kristie Miller

kristie.miller@sydney.edu.au

1 Department of Philosophy, University of Western Australia, Perth, Australia

2 Department of Philosophy, University of Sydney, Sydney, Australia

3 Department of Philosophy, University of Nottingham, Nottingham, UK 


\section{Introduction}

How do grounding and causation differ? One answer to this question appeals to time. Williamson, for instance, maintains that there is no grounding over time.

The challenger may argue that an object needs non-modal properties to ground its modal properties. For instance, a lump of clay is malleable (a modal property) because it has a certain microphysical structure (a non-modal property). The analogous principle in the temporal case is obviously false: what I was yesterday is not grounded in what I am today, in any useful sense. Even in the modal case, the principle is not obviously true. Indeed, its very meaning is unclear. What is the supposed distinction between 'modal' and 'non-modal' properties? Malleability is easy to put on the 'modal' side, because the word has the modal suffix '-ability'. But we cannot assume the mere absence of explicit modal indicators in the phrase 'microphysical structure' sufficient for the corresponding properties to be 'non-modal'. Presumably, the microphysical structure has consequences for what the object can or cannot do or have done to it, otherwise it would not ground malleability. So why exactly does microphysical structure count as 'nonmodal'? Perhaps the question has a good answer, but that too is to be found by theoretical enquiry. We cannot rely on untutored common sense alone.

(Williamson 2013, pp. 13-14). Similarly, Loewer (2012) defends a view according to which all metaphysical explanation — of which grounding would be an instanceis co-temporal (assuming that the explanandum and explanans are temporal), and to be contrasted with scientific explanation. As he has it:

Metaphysical explanation need not involve laws and the explanandum and explanans must be co-temporal (if the explanans is a temporal fact or property). Scientific explanation of a particular event or fact need not show that it grounded in a more fundamental event or fact but rather, typically, shows why the event occurred in terms of prior events and laws (Loewer 2012: 131).

Causation, of course, does occur over time. Call something in this spirit the 'temporal criterion' for differentiating cause from ground. ${ }^{1}$

Against this criterion, some philosophers have defended diachronic grounding. Baron (2015), for instance, maintains that present entities can ground the existence of past entities. In a similar vein, Wilson (forthcoming) has argued that present entities can be grounded in their causal histories. Tognazzini (2015) has defended an account of free will by appealing to diachronic grounding and Hazlett $(2006,2011)$ and Bennett (2017) also appear friendly to the idea that there can be grounding over time.

\footnotetext{
1 The temporal criterion is defended explicitly by Leuenberger (2013), assumed in some form by Bernstein (2016) and is in the background of Schaffer's $(2009,2016)$ work. Indeed, Schaffer (2016, p. 94) notes that grounding and causation might be a species of a single relation that is " "called 'causation' when it drives the world through time, and 'grounding' when it drives the world up levels". This distinction seems to assume some version of the temporal criterion. Wilson (forthcoming) is sceptical of the temporal criterion, presumably because Wilson (2018) takes grounding to be a kind of metaphysical causation.
} 
In this paper, we situate the temporal criterion within a broader framework that focuses on two aspects: locational overlapping in space and time and the presence of either grounding or causal intermediaries in space and time. We consider, and reject, the idea that the difference between grounding and causation is that grounding can occur without intermediaries. We go on to use the fact that grounding and causation both require intermediaries to develop a better temporal criterion for distinguishing causation from grounding. The criterion is this: when a cause and effect are spatially disjoint, there must be a chain of causal intermediaries between the cause and the effect that are extended in time. By contrast, when the grounds and the grounded are spatially disjoint, there must be a chain of grounding intermediaries, but the chain need not be extended in time, it can be purely spatial. The difference between grounding and causation, then, is that causation requires time for chaining in a way that grounding does not.

We begin, in Section Two, by setting up our framework by differentiating between locational overlapping and mediation for grounding and causation. We show that the standard temporal criterion can be reformulated within this broader framework, and that a spatial analogue can also be formulated. In Section Three, we go on to focus on one of these alternatives: the idea that grounding, and not causation, involves a kind of action at a distance: cases in which the grounds and the grounded are located at disjoint regions, and there is no chain of locationally overlapping grounding intermediaries. We reject this criterion and use the apparent need for grounding intermediaries as a basis for formulating our new temporal criterion in Section Four. We then consider the question of why the difference between grounding and causation we have identified should be given any metaphysical weight in differentiating the two notions.

\section{Location, location, location}

In what follows we will assume that grounding is an asymmetric, irreflexive, transitive and hyperintensional relation of relative fundamentality that introduces an ontological hierarchy amongst the things thus related. ${ }^{2}$ The relata of grounding relations are commonly taken to be facts, conceived as worldly entities comprised of objects, properties, and relations, in a structured manner. ${ }^{3}$ For simplicity, in this paper, we will simply talk of entities as the relata of grounding, where these can be construed as facts, or as objects, or as states of affairs. But nothing we say hangs on this choice.

We will say that an entity $\mathrm{x}$ wholly grounds an entity $\mathrm{y}$, when $\mathrm{x}$ is sufficient, on its own, to ground $\mathrm{y}$. We will also say that $\mathrm{x}$ partly grounds an entity $\mathrm{y}$ when $\mathrm{x}$ is a

\footnotetext{
${ }^{2}$ This is the orthodox view [Schaffer (2009); Trogdon (2013)]. Nevertheless, Schaffer (2012) questions the transitivity of partial grounding [see Litland (2013) for a response], Jenkins (2011) argues that grounding is merely quasi-irreflexive, Rodriguez-Pereyra (2015) argues that grounding is neither irreflexive, asymmetric nor transitive, and Duncan et al. (2017) argue that it's not hyperintensional.

3 See Audi (2012) and Raven (2012). This view is not universal; see, for instance, Schaffer (2009) for the view that the relata of grounding include a more liberal range of kinds of entities.
} 
member of some collection $\mathrm{x}_{1}, \ldots, \mathrm{x}_{\mathrm{n}}$ such that the $\mathrm{xn}$ together are sufficient to ground $y$. We will symbolise grounding quite generally using the '/ symbol and so ' $\mathrm{x} / \mathrm{y}$ ' means that $\mathrm{x}$ grounds $\mathrm{y}$ either in part or in whole.

Our framework for developing criteria for differentiating grounding from causation involves two dimensions: location and mediation. We take each in turn. We will assume that exact location $\mathrm{L}(\mathrm{x}, \mathrm{y})$ is the primitive location relation. Following Gilmore (2013), we adopt the following informal gloss on what it is for something to be exactly located:

Exact Location: An entity $\mathrm{x}$ is exactly located at a region $\mathrm{y}$ if and only if $\mathrm{x}$ has (or has-at-y) exactly the same shape and size as y and stands (or stands-at-y) in all the same spatial or spatiotemporal relations to other entities as does $y$.

We can use exact location plus the mereological relation of overlap to define a notion of locational overlap for grounding, along with its converse relation. Locational overlap occurs, roughly, when grounds and grounded share a location. The converse-the failure of overlap-occurs when the grounds are located at a region that is disjoint from the region at which the grounded is located.

There are two kinds of case to consider. The first kind of case is one in which two entities share a spatial location, but are temporally disjoint. The second kind of case is one in which two entities share a temporal location, but are spatially disjoint. One way to handle this distinction is to split overlap for regions into temporal and spatial forms. For this we will introduce two notions of overlap: S and T (for spatial and temporal regions respectively). A spatial region is just a set of values for the spatial coordinates $\{\mathrm{x}, \mathrm{y}, \mathrm{z}\}$; and a temporal region is just a value for the temporal coordinate $\{t\}$. To say that two regions spatially overlap is just to say that there is some overlap in their spatial coordinates, but not necessarily their temporal coordinates. ${ }^{4}$ To say that two regions temporally overlap, is to say that there is some overlap in their temporal coordinates, but not necessarily their spatial coordinates. $\mathrm{O}$ picks out neutral overlap, where there could be overlap in either the spatial or the temporal coordinates. We can then informally define temporally disjoint grounding for a case in which $\mathrm{x}$ grounds $\mathrm{y}$ as follows: $\mathrm{x}$ grounds $\mathrm{y}$ and there exist two distinct, non-temporally-overlapping locations, $\mathrm{z}$ and $\mathrm{w}$, such that $\mathrm{x}$ grounds $\mathrm{y}$, and $\mathrm{x}$ is exactly located at $\mathrm{z}$, and $\mathrm{y}$ is exactly located at $\mathrm{w}$.

\footnotetext{
${ }^{4}$ Where $\mathrm{O}$ is mereological overlap, and can be defined in terms of having a part, $\mathrm{P}$, in common as follows:

$$
O x y \leftrightarrow \exists z(P z x \& P z y)
$$

We can then define spatial and temporal overlap by introducing the notions of a spatial part, $\mathrm{P}_{\mathrm{s}}$, and a temporal part, $\mathrm{P}_{\mathrm{t}}$. A spatial part is a part that is located at a particular spatial region, $\mathrm{r}_{\mathrm{s}}$, and a temporal part is a part that is located at a particular temporal region, $\mathrm{r}_{\mathrm{t}}$. Spatial and temporal overlap can then be defined as follows:
}

$$
\begin{aligned}
& S x y \leftrightarrow \exists z\left(P_{S} z x \& P_{S} z y\right) \\
& T x y \leftrightarrow \exists z\left(P_{T} z x \& P_{T} z y\right) .
\end{aligned}
$$


Temporally disjoint grounding $={ }_{\text {df }}(x / y \& \exists z \exists w[L(x, z) \& L(y, w) \& \sim T(z, w)]$

And we can define spatially disjoint grounding in a similar way:

$$
\text { Spatially disjoint grounding }={ }_{\mathrm{df}}(x / y \& \exists z \exists w[L(x, z) \& L(y, w) \& \sim S(z, w)]
$$

It is also possible to combine these definitions to handle a case of spatially and temporally disjoint grounding. This would be a case in which $\mathrm{x}$ grounds $\mathrm{y}$ despite the fact that $\mathrm{x}$ and $\mathrm{y}$ share neither spatial nor temporal locations. We can define this simply in terms of the lack of overlap of any kind between the two locations at which $\mathrm{x}$ and $\mathrm{y}$ are located. ${ }^{5}$

$$
\text { Doubly Disjoint Grounding }={ }_{\text {df }}(x / y \& \exists z \exists w[L(x, z) \& L(y, w) \& \sim O(z, w)]
$$

Obviously, we can formulate a similar range of definitions for causation (these definitions are straightforward, and so we leave this as an exercise for the reader). Using the notion of locational overlap for grounding and causation, we can reformulate the standard temporal criterion for differentiating grounding from causation straightforwardly. On this formulation grounding is never temporally disjoint, but causation is.

As briefly mentioned, however, the temporal criterion is in trouble. As Wilson (forthcoming) has argued, there appear to be a number of cases of temporally disjoint grounding. Most of these involve entities being grounded in their causal histories. For example, suppose that part of what it is to be a church is to be consecrated. Now, consider a particular church-St. Brigid's. St. Brigid's is a church only if there was a time at which the relevant rights and rituals were performed on a particular building. We can thus say that the present fact that St. Brigid's is a church is partly grounded in the fact that the building of which it is partly constituted underwent consecration at some past time. Because the temporal location of the consecration is disjoint from the current temporal location of the church, this prima facie counts as a putative case of temporally disjoint grounding.

Or consider the fact that Alastair is human. If one accepts the essentiality of origin, then what it is to be human is to be descended from a particular lineage, which includes Homo Sapiens. Thus, swamp-person is not human because swampperson was created ex nihilo from the swamp, without an evolutionary history that connects swamp person to the Homo Sapiens lineage. The fact that Alastair is a

\footnotetext{
5 The definition of spatiotemporally disjoint grounding is similar to the definition of doubly disjoint grounding just outlined. In a spatiotemporal context, however, spatial and temporal distances must be replaced with spacelike and timelike distances. Thus, grounding at a temporal distance becomes grounding at a timelike distance and grounding at a spatial distance becomes grounding at a spacelike distance. We can define these two notions in terms of spacelike and timelike distance relations respectively. We can use $\mathrm{S}(\mathrm{x}, \mathrm{y})$ for ' $\mathrm{x}$ is at a spacelike distance from $\mathrm{y}$ ', which amounts to $\mathrm{x}$ and $\mathrm{y}$ having a positive spatiotemporal distance from one another in spacetime. We will use $T(x, y)$ for ' $x$ is at a timelike distance from $\mathrm{y}$ ', which amounts to $\mathrm{x}$ and $\mathrm{y}$ being at a negative spatiotemporal distance from one another. We can then replace the definitions of spatially and temporally disjoint grounding with the following pair of definitions:

Timelike Disjoint Grounding $={ }_{\mathrm{df}}(x / y \& \exists z \exists w[L(x, z) \& L(y, w) \& \sim O(z, w) \& S(z, w)]$ Spacelike Disjoint Grounding $=$ df $(x / y \& \exists z \exists w \exists v[L(x, z) \& L(y, w) \& \sim O(z, w) \& T(z, w)]$.
} 
human now is therefore grounded in some past events: the past events that constitute the right ancestral connection for membership in the kind Homo Sapiens. Again, because these facts about Alastair's heritage concern events in the past, it follows that Alastair's being human now is partly grounded in a past event. Since Alastair is located in the present, the grounding involved appears to be temporally disjoint grounding.

Wilson goes on to argue that what it is to be a past or future anything requires temporally disjoint grounding. For example, suppose that Sara is a retired dancer. Her possession of this property now, Wilson maintains, is grounded in facts about the past: namely, Sara's past activities as a dancer. Similarly, suppose that Hilary is a future president. Her possession of this property now is grounded in facts about the future: namely Hilary's eventual inauguration. Indeed, as Wilson notes, being the 'once and future king' is a property that Arthur possesses, but a property that is itself grounded in past and future facts about Arthur. All of these cases appear to be cases in which some property that is possessed by a person in the present, and which is located in the present, is grounded in properties or facts that are located at other times.

So the temporal criterion fails. Using the notion of locational overlap, however, we can modify the temporal criterion by turning it into a spatial requirement. Thus, one can say that the difference between grounding and causation is that causation is spatially disjoint, whereas grounding is not. Grounding is always spatially overlapping. Call this: the spatial criterion. The spatial criterion is interesting to consider because, to date, the focus has been on whether there can be grounding over time, and comparatively little consideration has been given to whether there can be grounding over space. Clearly, causation can occur over space, and so there is scope here to pull the two notions apart. Moreover, there is at least some initial plausibility to the idea that grounding is not spatially disjoint. After all, the location of the labradoodle is not disjoint from the location of the dog; the location of light blueness is not disjoint from the location of blueness; the location of the bachelor is not disjoint from the location of the unmarried man. And so on.

Ultimately, however, the spatial criterion also fails. In addition to cases of temporally disjoint grounding, there appear to be analogous cases of spatially disjoint grounding. Here are three such cases. First, consider Pluto. Pluto, we will suppose (perhaps controversially) is a planet. The fact that Pluto is a planet is partly grounded in conventions. It is we (or rather the International Astronomical Union) who decide whether or not Pluto has the property of being a planet. If we focus on a particular time at which Pluto is a planet, it's being a planet at that time is grounded in our conventions. Supposing that the conventions are located wherever the social system in which those conventions arise is located, it follows that Pluto, out there in space, is partly grounded at a time in the conventions that exist here. Note, of course, that this is not the crazy view that the hunk of rock that constitutes Pluto is grounded in what exists here. Rather, our claim is that Pluto qua planet appears to be partly grounded in the hunk of rock, and partly grounded in our conventions. This is spatially disjoint grounding because the location of the conventions and the location of the planet are disjoint. 
Second case: Koslicki (2015, p. 309) cites, as a case of grounding, a piece of Swiss cheese and its holes. The holes in the cheese are grounded in the piece of Swiss cheese itself. Plausibly, however, there is no cheese where the holes are located, and no holes where there is any cheese. ${ }^{6}$ Given that the cheese constitutes the boundaries of any given hole, it seems we have a case in which the holes are grounded in the boundary, but where the boundary and the holes occupy disjoint spatial locations, at a time. This, like the planet case, appears to be a case of spatially disjoint grounding.

Third example: the flagpole and its shadow. Suppose that we have a flagpole, and that the flagpole casts a particular shadow. The flagpole on its own does not ground the shadow, but it certainly seems to be a part of the grounding basis for the shadow. It is, plausibly, the flagpole plus the laws of nature-in particular, the laws governing the rectilinear propagation of light-plus the presence of a light source shining on the flagpole that, together, ground the existence of the shadow. This case of grounding happens at a time. For any given time at which a shadow is cast, the shadow is grounded, in part, in the flagpole. However, the flagpole and the shadow occupy disjoint spatial locations. Everywhere that the flagpole is, the shadow isn't.

We will return to the above cases of spatially and temporally disjoint grounding in the next section. For now it is enough to see that locational overlap on its own is not sufficient to pull grounding and causation apart. Locational overlap needs to be supplemented with something else. One natural suggestion is to supplement locational overlap with a notion of mediation: chains of grounding that connect the grounds to the grounded, when the grounds and the grounded are locationally disjoint.

To see the idea, we will start by defining direct and indirect grounding as follows:

$$
\begin{aligned}
& \text { Direct grounding }=\text { df }[x / y \& \sim \exists z(x / z \& z / y)] \\
& \text { Indirect grounding }={ }_{\text {df }}[x / y \& \exists z(x / z \& z / y)]
\end{aligned}
$$

We will adopt the symbol ' $/ \mathrm{D}$ ' for cases of direct grounding and the symbol ' $/ \mathrm{I}$ ' for cases of indirect grounding. We can use the combination of direct and indirect grounding to characterise a grounding chain. Thus we will say that $\mathrm{x} / \mathrm{I} \mathrm{y}$ via a grounding chain when for a sequence $\mathrm{z}_{1} \ldots \mathrm{z}_{\mathrm{n}}$ such that $\mathrm{z}_{1} / \mathrm{D}_{\mathrm{D}} \mathrm{z}_{2}, \mathrm{z}_{2} / \mathrm{D}_{\mathrm{D}} \mathrm{z}_{3} \ldots \mathrm{z}_{\mathrm{n}-1} / \mathrm{D}_{\mathrm{D}} \mathrm{z}_{\mathrm{n}}$, $\mathrm{x}=\mathrm{z}_{1}$ and $\mathrm{y}=\mathrm{z}_{\mathrm{n}}$. We can then use the notion of a grounding chain to define a locationally overlapping grounding chain. Thus, we will say that $\mathrm{x} / \mathrm{I}$ y via a locationally overlapping grounding chain when $\mathrm{x} / \mathrm{I} \mathrm{y}$ via a grounding chain, and for locations $r_{1} \ldots r_{n}$ such that for each successive pair of members $<z_{n}, z_{n+1}>$ in the grounding chain $\mathrm{L}\left(\mathrm{z}_{\mathrm{n}}, \mathrm{r}_{\mathrm{n}}\right)$ and $\mathrm{L}\left(\mathrm{z}_{\mathrm{n}+1}, \mathrm{r}_{\mathrm{n}+1}\right), \mathrm{r}_{\mathrm{n}}$ and $\mathrm{r}_{\mathrm{n}+1}$ overlap (i.e. $\mathrm{O}\left(\mathrm{r}_{\mathrm{n}}, \mathrm{r}_{\mathrm{n}+1}\right)$ ).

Using the notion of a locationally overlapping grounding chain we can define a notion of 'action at a distance' for grounding (henceforth: just 'grounding at a distance'). This is a case in which the grounds and the grounded are locationally disjoint and there is no locationally overlapping grounding chain connecting them. This is analogous to a notion of causal action at a distance, which involves any case in

\footnotetext{
6 This is on the assumption that something like the Lewis and Lewis (1970) view of holes is right.
} 
which the cause and the effect are located at some spatial distance from one another, and there is no chain of intermediaries between the cause and the effect such that each link in the chain involves a locationally overlapping cause and effect pair.

We can informally define grounding at a distance for a case in which $\mathrm{x}$ grounds $\mathrm{y}$ as follows: $x$ grounds $y$ and there exist two distinct, non-overlapping locations, $\mathrm{z}$ and $\mathrm{w}$, such that $\mathrm{x}$ is located at $\mathrm{z}$ and $\mathrm{y}$ is located at $\mathrm{w}$ and either $\mathrm{x}$ directly grounds $\mathrm{y}$ or $\mathrm{x}$ indirectly grounds $\mathrm{y}$ and there is no locationally overlapping grounding chain between $x$ and $y$. If we introduce a three-place predicate $\operatorname{GCL}(x, y, z)$ for: $x$ grounds $y$ via a locationally overlapping chain $z$, then we can provide formal definitions of grounding at a temporal and spatial distance as follows:

Grounding at a temporal distance

$$
={ }_{\mathrm{df}}\left(x / y \& \exists z \exists w[L(x, z) \& L(y, w) \& \sim T(z, w)] \&\left(x /{ }_{D} y \vee\left(x /{ }_{I} y \& \sim \exists t[\operatorname{GCL}(\mathrm{x}, \mathrm{t}, \mathrm{y})]\right)\right)\right)
$$

Grounding at a spatial distance

$$
={ }_{\mathrm{df}}\left(x / y \& \exists z \exists w[L(x, z) \& L(y, w) \& \sim S(z, w)] \&\left(x /{ }_{D} y \vee\left(x /{ }_{I} y \& \sim \exists t[\operatorname{GCL}(\mathrm{x}, \mathrm{t}, \mathrm{y})]\right)\right)\right)
$$

It is also possible to combine these definitions to handle a case of grounding where $\mathrm{x}$ grounds $\mathrm{y}$ despite the fact that $\mathrm{x}$ and $\mathrm{y}$ share neither spatial nor temporal locations. We can define this simply in terms of the lack of overlap between the two locations at which $\mathrm{x}$ and $\mathrm{y}$ are located. ${ }^{7}$

Grounding at a double distance

$$
={ }_{\mathrm{df}}\left(x / y \& \exists z \exists w[L(x, z) \& L(y, w) \& \sim O(z, w)] \&\left(x /{ }_{D} y \vee\left(x /{ }_{I} y \& \sim \exists t[\operatorname{GCL}(\mathrm{x}, \mathrm{t}, \mathrm{y})]\right)\right)\right)
$$

The notion of grounding at a distance can be used to formulate a new way of differentiating grounding from causation. It is often thought that causation requires a causal intermediary between any cause-whole or partial-and its effect, if the cause and effect are not spatially or temporally adjacent to one another. Causation cannot 'jump' across time or space; there is no causation at a distance. ${ }^{8}$

By contrast, one might think that there can be grounding at a distance. Indeed, it might be thought that each of the cases of locationally disjoint grounding described above-disjoint in space or time_-are also cases of grounding at a distance. So, for instance, suppose, once again, that some event in the past-a consecration act-is a

\footnotetext{
7 We can then modify our definitions to be compatible with spacetime, in the same manner as above. Grounding at a timelike distance$$
={ }_{\mathrm{df}}\left(x / y \& \exists z \exists w[L(x, z) \& L(y, w) \& \sim O(z, w) \& S(z, w)] \&\left(x /{ }_{D} y \vee\left(x /{ }_{I} y \& \sim \exists t[\operatorname{GCL}(\mathrm{x}, \mathrm{t}, \mathrm{y})]\right)\right)\right)
$$$$
\text { Grounding at a spacelike distance }
$$$$
={ }_{\mathrm{df}}\left(x / y \& \exists z \exists w[L(x, z) \& L(y, w) \& \sim O(z, w) \& T(z, w)] \&\left(x /{ }_{D} y \vee\left(x /{ }_{I} y \& \sim \exists t[\operatorname{GCL}(\mathrm{x}, \mathrm{t}, \mathrm{y})]\right)\right)\right),
$$

8 As we shall see below, the notion of causation at a distance that we have in mind is what Frisch (2010, p. 657) calls causal non-locality, and which Hall (2002, p. 276) defines as follows:
}

We have a case of [causal non-locality] if we have a cause, at least one of whose effects is not connected to it via any spatiotemporal continuous causal chain.

Causal locality is thus the requirement that causes be related to effects via spatiotemporal continuous causal chains. 
partial ground for the current existence of a church. It could be argued that there does not need to be a grounding intermediary between that consecration act and the current church in order for the grounding to obtain. Grounding can not only occur across time, but it can also jump across time. Similarly, in the Pluto case, one might think that the conventions on Earth that ground Pluto's planethood do so despite the absence of a grounding intermediary between the two. Grounding, in the case of Pluto's planethood, jumps across space.

Call this: the action at a distance criterion for differentiating causation from grounding. We think that this criterion also fails, but that its failure is instructive: it helps us to develop a viable account of the difference between causation and grounding, one that can be plausibly thought of as the spiritual successor to the temporal criterion. In order to show this, we will consider the action at a distance criterion in the next section. We will proceed in two stages. First, we will show that all cases of apparent grounding at a distance can be naturally reinterpreted in such a way that there is something mediating the grounding. Second, we will consider what it would take to have genuine grounding at a distance and suggest that the relevant conditions are unlikely to obtain. After that, we will present our favoured criterion for differentiating causation from grounding that takes the lack of grounding at a distance as its starting point.

\section{Grounding at a distance}

Consider Pluto once again. What we aim to ground in this case is Pluto's being a planet. Note, first, that Pluto exists at a particular spatial location, $\mathrm{R}_{1}$. Notice, further, that Pluto's being a planet is not intrinsic to Pluto. For it to be the case that being a planet is intrinsic to Pluto, it must be the case that any duplicate of Pluto is also a planet. We can, however, imagine duplicating Pluto without duplicating Pluto's planethood. That's because we can imagine duplicating Pluto without duplicating the conventions laid down by the IAU regarding what it takes for something to be a planet. If we were to put Pluto into a world in which the conventions surrounding planethood are different, it would follow that in that world Pluto is not a planet, despite being intrinsically the same as it is actually.

Assuming that being a planet is an extrinsic feature of Pluto, Pluto's being a planet will be grounded in a relation between Pluto and a group of conventions laid down by the IAU on Earth. The relation in question is the relation of satisfaction. The satisfaction relation itself is located wherever its relata are located. And so the satisfaction relation extends between Pluto, located at $\mathrm{R}_{1}$, and the conventions, located at $R_{2}$. What grounds the fact that Pluto stands in the satisfaction relation is just that Pluto possesses a range of properties - it has a certain size, shape, and so on-and because it possesses these properties it satisfies the conventions laid down by the IAU for being a planet.

So, on this way of understanding the picture, properly speaking Pluto's being a planet at $R_{1}$ is not grounded in the conventions laid down by the IAU at $R_{2}$. Rather, Pluto's being a planet at $R_{1}$ is grounded in the satisfaction relation between Pluto and the conventions in question. Since the satisfaction relation is located at $R_{1}$ and 
$\mathrm{R}_{2}$ (and in between), the relation of satisfaction acts as a grounding intermediary and so the grounding is not at a spatial distance. Of course, there is still grounding at spatially disjoint regions: it is still the case that the conventions on Earth partially ground Plato's planethood. It's just that the conventions do so by transitivity, and via the fact that the satisfaction relation acts as an intermediary.

This analysis of the Pluto case suggests a general strategy for handling putative cases of grounding at a spatial distance. First, we find a property $\mathrm{P}$, such as being $a$ planet, that appears to be partially grounded at a spatial distance by $\mathrm{Q}$. We then investigate $\mathrm{P}$ (in this case, being a planet). We attempt to argue that $\mathrm{P}$ is not directly grounded by $\mathrm{Q}$, but is directly grounded by some $\mathrm{R}$, connecting $\mathrm{P}$ and $\mathrm{Q}$ (in the case of Pluto we argued for this by arguing that Pluto is only a planet because of a relation that relates Pluto and some conventions on the Earth. This relation, we said, is what ground's Pluto's being a planet). But, because $\mathrm{R}$ is located across those spatial regions occupied by $\mathrm{P}$ and $\mathrm{Q}$, it then follows that we do not have an instance of P's being directly grounded by $\mathrm{Q}$ at a spatial distance. Rather, we have an instance of $\mathrm{P}$ being directly grounded by $\mathrm{R}$, where $\mathrm{R}$ overlaps both $\mathrm{P}$ and $\mathrm{Q}$ and stretches between them, and so acts as an intermediary.

With this broad strategy in mind, return to the case of a hole and its boundary. One way to handle this case is to treat 'being a hole' as an extrinsic feature of a region. To say that there is a hole in the cheese is just to say that there is a region that is related to the cheese in the right way. In particular, the region is related to the cheese by the relation of 'being bounded by'. Thus the region is a hole because it is bounded by the cheese. As with the planet case, this seems plausible because we can imagine duplicating the region without duplicating the hole. For instance, we could duplicate the region that is bounded by the cheese, without duplicating the cheese itself. In this situation we would have failed to duplicate the hole despite the fact that we have duplicated the region. The relation 'being bounded by' is extended across the region occupied by the boundary, $\mathrm{R}_{2}$, and the region occupied by the hole, $\mathrm{R}_{1}$, in virtue of having relata at both of those regions. $\mathrm{R}_{1}$ itself stands in the 'being bounded by' relation simply in virtue of the fact that it has a particular size, shape and spatial/temporal properties that mean it is located where it is relative to other regions. The fact that $R_{1}$ is a hole is thus grounded in a relation in which $R_{1}$ stands. Since the relation is extended across $R_{1}$ and $R_{2}$, the grounds are not disjoint from the grounded and so there is no grounding at a distance in this case.

Of course, one might be tempted to think that one cannot duplicate the hole without duplicating both the cheesy boundary and the region $\mathrm{R}_{1}$. But then the right thing to say is that the hole is an object that itself is made up of the region $R_{1}$ and the boundary of cheese located at $R_{2}$, which encloses $R_{1}$. The hole is grounded at those regions simply in virtue of having two parts-one located at $R_{2}$ and one located at $\mathrm{R}_{1}$ - which ground the hole. ${ }^{9}$ But note that if that story is correct, then none of the grounding is at a distance: the hole is partly grounded at $R_{1}$ by $R_{1}$, and is partly grounded at $R_{2}$ by the cheesy boundary located there. The failure of locational

\footnotetext{
9 Something like this view is defended by Casati and Varzi (1994) and Miller (2007).
} 
overlapping is essential for grounding at a distance, and there is no such failure in this case.

The flagpole and shadow case can be handled in much the same way. First, we can identify a property - the property of being a shadow-which is possessed by a particular region $\mathrm{R}_{1}$. As before, we ground the property of being a shadow in a relation; this time the relation of being shaded by that obtains between the flagpole and the region $\mathrm{R}_{1}$. The fact that $\mathrm{R}_{1}$ appears to have the property being a shadow might seem like an intrinsic property of $R_{1}$. However, we can easily duplicate $R_{1}$ without duplicating the shadow. The shadow itself is grounded in the property of being a shadow possessed by $\mathrm{R}_{1}$, which is, in turn, grounded in the relation being shaded by that stands between the flagpole, the light source, and $\mathrm{R}_{1} \cdot \mathrm{R}_{1}$ 's standing in this relation is due to the intrinsic properties of $\mathrm{R}_{1}$ : it's size, shape and location. Since the relation itself is located both at $\mathrm{R}_{1}$, and where the light-source and flagpole are located, this is not a case of grounding at a distance.

Having seen how the strategy works in the spatial case, we can now apply it to the temporal case. It's easy to see how the story will go. We find a property, P, that appears to be directly grounded at a temporal distance by some $\mathrm{Q}$. We then investigate $\mathrm{P}$. We attempt to argue that $\mathrm{P}$ is not directly grounded by $\mathrm{Q}$, but by some $R$ connecting $\mathrm{P}$ and $\mathrm{Q}$. But, because $\mathrm{R}$ is located across those temporal regions occupied by both $\mathrm{P}$ and $\mathrm{Q}$, it then follows that we do not have a case of P's being directly grounded by $\mathrm{Q}$ at a temporal distance. Rather, we have an instance of $\mathrm{P}$ being directly grounded by $\mathrm{R}$, where $\mathrm{R}$ overlaps $\mathrm{P}$ and $\mathrm{Q}$.

The case of St. Bridget's consecration can be handled using this broad strategy. We find a property $\mathrm{P}$-such as being a church-that appears to be directly grounded at a temporal distance by $\mathrm{Q}$ (the past act of consecration). We then investigate $\mathrm{P}$ (in this case, being a church). We then show that $\mathrm{P}$ is not directly grounded by $\mathrm{Q}$ at all, but rather, is directly grounded by some $\mathrm{R}$, connecting $\mathrm{P}$ and $\mathrm{Q}$ (in the case of St Brigid's this is the relation of the satisfaction of the conventions around consecration). But, because $R$ is located across the regions occupied by $\mathrm{P}$ and $\mathrm{Q}$, it follows that we do not have an instance of $P$ 's being directly grounded by $\mathrm{Q}$ at a temporal distance. Rather, we have an instance of P's being directly grounded by $\mathrm{R}$, where $\mathrm{R}$ overlaps both $\mathrm{P}$ and $\mathrm{Q} .{ }^{10}$

The case of Alastair's humanity is slightly different if we allow that being human is not a matter of satisfying a convention. Instead, then, we should say that Alastair's being human is grounded in the causal chain that connects him to the ancestral lineage Homo Sapiens. In this case, the property of being human is grounded in a temporally extended property (being a part of a causal chain that connects Alastair to past humans) that relates Alastair to the past humans who

\footnotetext{
10 Alternatively, we could think that that there is a series of grounding intermediaries between $\mathrm{P}$ and $\mathrm{Q}$, not just R: namely the persisting church which, at each time it exists, is grounded by a building and some conventions that exist at that very time. The exact details will vary here depending on whether one thinks of the building and conventions as enduring or perduring, but the crucial details remain the same. Both the building and the conventions persist. So there is a series of grounding intermediaries between $\mathrm{P}$ and $\mathrm{Q}$ : the persisting building and the persisting conventions that stretch between $\mathrm{P}$, all the way back to $\mathrm{Q}$. Hence there is no grounding at a distance.
} 
caused Alastair to come into existence who, in turn, are causally related to past humans, all the way back into the full ancestral lineage. A part of that chain is present - the part that has Alastair's present existence as the last relata in a series of causal relations. But, again, the grounding in this case is not at a distance. Alastair's possession of the property of being human is grounded in the relevant causal chain, which consists in a series of causal relations. And swamp-person is not human because they do not stand in the right causal relations. None of this requires it to be the case that Alastair's humanity is grounded at a distance in the past. Simply put, the causal relations themselves act as grounding intermediaries.

What about the case of Sara the retired dancer? Or the once and future king? We'll use the case of Sara as a stalking horse. Being a retired dancer is a relational property. It is to be understood as a coming together of Sara not being a professional dancer (for she must not have come out of retirement) as well as the right causal chain linking her current self with her earlier professional-dancer self. One relatum is the present and not-dancing Sara, and the other relatum is a past Sara who has gone through the process of retirement (whatever that involves). To say that Sara is a retired dancer is just to say that she stands in the relevant causal relation to the past. The relation itself extends across time, and is partly grounded in Sara now and Sara in the past. As was the case with Alastair's humanity, Sara's being a retired dancer is grounded in this particular causal chain, which mediates the grounding between present and past entities, a part of which is itself present. At least in and of themselves, then, it appears that these cases present no particular challenge to our recipe for translating apparent cases of grounding at a distance into cases of ordinary, mediated grounding. ${ }^{11}$

So far, we have argued that cases of what appear to be grounding at a distance can be reinterpreted. Of course, this does not show that grounding at a distance doesn't as a matter of fact happen. The point is simply that we don't, yet, have evidence that it does. In the cases described we can tell the complete story of grounding without appealing to grounding at a distance and that tends to cast doubt on the claim that there is any. Still, we think there are reasons to embrace the stronger conclusion that grounding is always mediated.

Very broadly, the reinterpretation strategy we have employed in the previous section involves taking some apparently intrinsic feature of an entity which appears to be directly grounded in the intrinsic features of some other entity at a disjoint location, and showing that the grounding can be reimagined in broadly extrinsic terms. So, for instance, we took what seemed to be an intrinsic aspect of Pluto- that

\footnotetext{
11 What we have said thus far may seem to rest heavily on an eternalist metaphysics. We have said that there are entities and conventions that are located in the past, that there are relations with relata that are located at distinct times and so on. If one is a presentist, then these claims are false. Grounding at a temporal distance would require the existence of grounding relations that extend across time, and that take distinct objects at distinct times as relata. Presentists, however, typically deny that there are any relations of this kind. And so presentists may just have to deny that any of the above cases are genuine cases of grounding at a distance anyway. Or, if they don't deny this, then they are already tasked with accounting for these putative cases of grounding without appealing to grounding relations that connect objects at distinct temporal locations. Whatever the right presentist account of this turns out to be (if there is one) we will simply adopt that as part of the broad strategy we are employing here.
} 
it is a planet-and showed how planethood is not a purely intrinsic feature. Whether or not something is a planet partly depends on conventions that are located on Earth, as dictated by scientists and the IAU. Once we see that planethood is not purely intrinsic, we can then re-characterise the direct grounding of planethood in terms of entities that are located at the regions across which planethood as an extrinsic feature extends. Hence there is no grounding at a distance, since in fact the grounding proceeds via grounding intermediaries, and these intermediaries connect the grounds that are locationally disjoint from the grounded, to the location of the grounded.

In light of this, an unambiguous case of grounding at a distance would be one in which the intrinsic features that we are trying to ground cannot be recast in purely extrinsic terms. In particular, such a case would involve two entities, $\mathrm{E}_{1}$ (exactly located at region $\mathrm{R}_{1}$ ) and $\mathrm{E}_{2}$ (exactly located at disjoint region $\mathrm{R}_{2}$ ) with purely intrinsic features $\mathrm{P}$ and $\mathrm{Q}$ respectively, such that $\mathrm{E}_{1}$ 's being $\mathrm{P}$ at $\mathrm{R}_{1}$ directly grounds $E_{2}$ 's being $Q$ at $R_{2}$. Such a case would not be open to the reinterpretation strategy we have used, since the intrinsic features demand to be understood in intrinsic terms.

It is, however, difficult to see how there could be any such cases. The very notion of intrinsicality would seem to undermine the possibility. An intrinsic feature is supposed to be a feature that an entity has purely in virtue of itself and nothing else. When an entity, $\mathrm{E}_{1}$, has a feature that is directly grounded in the intrinsic features of another entity, $E_{2}$, located in a disjoint region of spacetime, it seems that the relevant feature is not had purely in virtue of how $E_{1}$ is. Rather, the feature is had in virtue of how $\mathrm{E}_{2}$ is. It is therefore quite difficult to see how the feature can claim to be genuinely intrinsic.

To this one might reply that intrinsic features are grounded all the time! What we have just said seems to suggest that no entity can ground the intrinsic features of another. However, it is plausible to suppose that the grounding of intrinsic features is constrained by distinctness in this sense: an entity $\mathrm{E}_{2}$ can directly ground the intrinsic features of $E_{1}$ only if $E_{2}$ and $E_{1}$ are not wholly distinct entities. So, for instance, a particular molecular structure can directly ground the intrinsic hardness of a diamond, but that's only because the diamond and its molecular structure are not wholly distinct entities. Similarly, the number 2 can directly ground an intrinsic feature of the set $\{2\}$ : namely, that $\{2\}$ is a singleton set. But sets are not wholly distinct from their members. They are, plausibly, constituted by their members in at least some sense.

In general, then, the grounding of intrinsic features seems to go hand in hand with the absence of distinctness. The problem, then, for grounding at a distance is that in order for it to be the case that $E_{1}$ and $E_{2}$ are not wholly distinct, $E_{1}$ and $E_{2}$ should be located at regions that wholly overlap. Or, at the very least, the degree to which $\mathrm{E}_{1}$ and $E_{2}$ fail to be wholly distinct should be a function of the degree to which the regions at which $E_{1}$ and $E_{2}$ are located overlap: the greater the overlap, the less distinct are $E_{1}$ and $E_{2}$. When an entity $E_{1}$ is exactly located at a region $R_{1}$, and an entity $E_{2}$ is exactly located at a region $R_{2}$ that is disjoint from $R_{1}, R_{1}$ and $R_{2}$ do not overlap at all. And so, the necessary condition for the failure of distinctness is not met. $E_{1}$ and $E_{2}$ appear to be wholly distinct entities. Given that they are wholly 
distinct, it is not at all clear how the intrinsic features of $\mathrm{E}_{1}$ can be directly grounded in the intrinsic features of $E_{2}$. Any feature of $E_{1}$ that gets grounded in $E_{2}$, would seem to be a case in which $E_{1}$ doesn't have that feature in virtue of itself, but in virtue of how a wholly distinct entity- $\mathrm{E}_{2}$ - is. And so, the feature just does not seem to be intrinsic. If the feature is not intrinsic, however, and if it is extrinsic, then we are confident that some version of the reinterpretation strategy will work. If that is so, then there is no reason that we can see to suppose that grounding ever occurs at a distance.

\section{A new temporal criterion}

So far we have seen how the failure of locational overlapping on its own cannot be used to differentiate causation and grounding. We have also seen how a simple criterion based on grounding at a distance fails to differentiate causation and grounding. The failure of the grounding at a distance criterion is, however, instructive. The central reason why the grounding at a distance criterion appears to fail is that grounding, like causation, is mediated. There does, however, appear to be a difference in the way that grounding and causation are mediated, in cases where the cause/grounds and effect/grounded are locationally disjoint.

Consider a cause, $\mathrm{C}$, located at a spatial region $\mathrm{S}_{1}$, and an effect, E, located at a spatial region $S_{2}$, such that $S_{1}$ and $S_{2}$ are disjoint regions. $C$ can cause $E$ but only if there is a chain of locationally overlapping intermediaries that link $\mathrm{C}$ to $\mathrm{E}$. It is very plausible to suppose, however, that in order to connect the two spatially disjoint regions, the causal chain must unfold in time. There cannot be a chain of causal intermediaries that is purely spatial connecting the two spatially disjoint regions. Indeed, chains of purely spatial causation of this kind are forbidden by the special theory of relativity. Very roughly, according to the special theory of relativity, if two events are spatially disjoint, then the only way for the two events to be causally related is if the two events are also temporally disjoint from one another. A chain of purely spatial causation such as this would require instantaneous causal processes, and thus would seem to require causal processes that are faster-than-light.

Of course, talk of 'space' and 'time' is a bit loose when we are thinking of special relativity and thus spacetime. Using the language of spacetime, the requirement is that there can be no causal relations between events that are spacelike separated. All causation occurs between timelike separated events. What this means, however, is that if we have an event that is on Earth, and another event that is on Mars, then the only way for these two events to be causally related to one another is if they are also separated in time. Any chaining that occurs between the events to prevent action at a distance must itself be extended through time, since we can apply the same reasoning to any spatially disjoint pair of events in the chain. ${ }^{12}$

\footnotetext{
12 The constraint we are referring to is the requirement that causation be local. Frisch $(2010$, p. 658) draws a distinction between two notions of locality that is relevant to the present discussion: causal locality (which is the notion we have been working with thus far) and nomic locality, which following Belot (1998, p. 540) can be defined as follows:
} 
To be clear, our claim is not that causation is never simultaneous, and that all causation requires time to occur (though this may be true). The point, rather, is that events that are spatially separated from one another cannot be chained by a chain of events that is not extended in time. This is a prohibition on simultaneous causation in some sense, but it is not a blanket ban. It may be possible to make sense of simultaneous causation as occurring between events that are not spatially separated from one another. When events are at some spatial distance, however, there needs to be a temporally extended causal chain between them

Grounding, so far as we know, is under no such restriction. Consider, again, the Pluto case, and consider the analysis of that case that we have offered above. According to this analysis, there are some conventions, located at a spatial region $S_{1}$, and there is a hunk of matter located at a spatial region $S_{2}$. The conventions and the matter, together, ground a satisfaction relation, which in turn grounds Pluto's planethood. In this situation, we can say that the conventions at $S_{1}$ ground Pluto's planethood at spatial region $S_{2}$ in virtue of the fact that there is a grounding intermediary that overlaps $S_{1}$ and $S_{2}$ - the satisfaction relation-and thus there is a locationally overlapping chain of grounding. Grounding, unlike causation, does not require time in order to chain.

We are now in a position to state a new temporal criterion:

The New Temporal Criterion (NTC) For any $\mathrm{x}$ and $\mathrm{y}$, if $\mathrm{x}$ and $\mathrm{y}$ are spatially disjoint then $\mathrm{x}$ causes $\mathrm{y}$ only if there is a locationally overlapping chain of causes from $\mathrm{x}$ to $\mathrm{y}$ that is extended in time. It is not the case that for any $\mathrm{x}$ and $\mathrm{y}$, if $\mathrm{x}$ and $\mathrm{y}$ are spatially disjoint then $\mathrm{x}$ grounds $\mathrm{y}$ only if there is a locationally overlapping chain of grounds from $\mathrm{x}$ to $\mathrm{y}$ that is extended in time.

The basic idea behind the criterion is that causation requires time in a way that grounding doesn't. And so while it is not the case that only causation occurs over time, there is still a substantial difference between grounding and causation when it comes to how the two relate to time. In this way the new temporal criterion succeeds the old one.

Of course, it is not enough to simply state the NTC. Some consideration must be give as to why we should take the NTC to represent a meaningful difference between causation and grounding, such that we have some reason to take them to be

\section{Footnote 12 continued}

(Nomic Locality) The laws governing a situation are local exactly if in order to predict what will happen here in a finite amount of time, $\Delta t$, we need only look at the present state of the world in a finite neighbourhood of here, and the size of the neighborhood shrinks to zero as $\Delta t \rightarrow 0$.

It could be argued that nomic locality is the kind of locality found in relativity. If one also argues that nomic locality does not imply causal locality, then the core of our argument fails, since the local character of relativity does not provide a basis for the locality of causation. There are two things to say here. First, as Frisch (2010, p. 664) argues, nomic locality does imply some version of causal locality in the context of a field-theoretic interpretation of general relativity, which is the standard approach. Second, it may be possible to reframe NTC below in terms of nomic locality. The idea would be that causation is nomically local, in the sense that spatially disjoint causes and effects have to be connected by a temporally extended chain of nomic dependencies. The same may not apply to spatially disjoint grounds and grounded, which don't have to be connected by a temporally extended chain of nomic dependencies in the same way. 
different relations, metaphysically speaking. To see the point more clearly, consider that there are many relations that fall under the broad banner of grounding: composition, constitution, realization, determination and more, can all be thought of as grounding relations. But, of course, there are differences between each of these relations. Composition is not the same thing as material constitution, and the determinate/determinable relation is different again. Nonetheless, many view these relations as having enough in common to consider them all to be cases of grounding. ${ }^{13}$ The question, then, is whether the difference between causation and grounding that we have identified is enough to prevent causation from being considered a form of grounding. If not, then the NTC doesn't really get us anywhere (we may as well have noted that grounding and causation differ in how they are labelled!)

We believe that the NTC identifies a metaphysically important difference between grounding and causation. A genuine difference between the two relationsone on which we might hang a distinction between grounding and causationshould, at a minimum, make a difference to how the two relations work. This is, indeed, the case. The NTC encodes the fact that causation cannot be chained across space, whereas grounding can. Because grounding is able to chain across space, it is able to give structure to the world by relating spatially disjoint entities that exist at different levels of fundamentality. This, in turn, allows grounding to 'govern' the entire world up the axis of fundamentality, by relating spatially disjoint entities at different levels via some intermediary. Causation, by contrast, cannot govern the entire world up the axis of fundamentality in the same manner, because it does not permit the same kind of chaining between spatially disjoint events. If there are any spatially disjoint events that are located at different levels of fundamentality, causation has to chain these two events over time.

Being able to structure the entire world in a purely spatial manner is an important part of the job description of grounding, and the way that grounding can chain spatially disjoint entities over space helps it play this role in theorising. It is notable, then, that grounding is able to chain over space in a way that causation cannot. This difference between the two relations is substantive precisely because it goes to the core of what grounding is supposed to do for us. That causation cannot do this suggests that there is an important metaphysical difference between the two notions, a difference that the NTC is tracking.

To be clear, the NTC is not telling us that grounding and causation differ in the following respect: only grounding can chain over space, causation cannot. There may be causal chaining between events that are located at the same place, where the chain is not extended in time. We cannot yet see a way to rule that out. The NTC tracks the weaker claim that only grounding can chain spatially disjoint events over space. The difference between causation and grounding is thus perhaps not as clean as one might like. It would be cleaner if we could rule out purely spatial chains of causation. For then we could simply say that the difference between causation and grounding is that only grounding can give structure to the entire world in a purely

13 Though there are exceptions. See Wilson (2014) and Koslicki (2015). 
spatial manner, since only grounding can give us purely spatial chains, which are needed to produce structure. ${ }^{14}$

\section{Conclusion}

The NTC locks onto an important difference between causation and grounding. Chaining spatially disjoint events over space enables grounding to do what it does best: structure the world in a purely spatial manner. That causation cannot do this is some reason to suppose that they are metaphysically different. We can expect the difference between grounding and causation identified to be relatively immune to counterexamples. In order to provide a counterexample to the criterion, one would need to show that there are chains of causation between spatially disjoint events and that the chains are not also extended in time. Such a thing would plausibly violate relativity, and so we can be confident that there won't be any cases like this that will come to light. It is, however, worth thinking about whether the NTC can be strengthened. To strengthen it we must rule out purely spatial chains of causation. Whether that can be done remains to be seen.

Acknowledgements Sam Baron's work on this paper was partly funded by an Australian Research Council Discovery Early Career Researcher Award DE180100414 and a Discovery Project DP180100105. Kristie Miller's work on this paper was partly funded by an Australian Research Council Discovery Project DP180100105.

Open Access This article is distributed under the terms of the Creative Commons Attribution 4.0 International License (http://creativecommons.org/licenses/by/4.0/), which permits unrestricted use, distribution, and reproduction in any medium, provided you give appropriate credit to the original author(s) and the source, provide a link to the Creative Commons license, and indicate if changes were made.

\section{References}

Audi, P. (2012). A clarification and defense of the notion of grounding. In F. Correia \& B. Schnieder (Eds.), Metaphysical grounding: Understanding the structure of reality (pp. 101-121). Cambridge: Cambridge University Press.

Baron, S. (2015). The Priority of the Now. Pacific Philosophical Quarterly, 96, 325-348.

\footnotetext{
14 One might worry that the NTC isn't a very useful criterion for differentiating grounding from causation. The trouble is that it cannot be used as a basis for telling whether we have a chain of grounding before us or a chain of causation, when we are looking at a temporally extended chain of some kind. But we don't think it's a problem that the criterion is not useful in this particular respect. That's because we don't need any help in identifying cases of grounding and telling them apart from cases of causation. We seem pretty capable of doing this without a criterion for differentiating between the two notions. What we want, rather, is a criterion that identifies a difference between grounding and causation that is plausibly an important metaphysical difference between the two. The criterion we have identified has at least some claim to being a criterion like this since, as we have noted, the fact that grounding can occur across space without the need for any chaining across time means that grounding can structure reality over space, whereas causation cannot.
} 
Belot, G. (1998). Understanding electromagnetism. British Journal for the Philosophy of Science, 49(4), $531-555$.

Bennett, K. (2017). Making things up. Oxford: Oxford University Press.

Bernstein, S. (2016). Grounding is not causation. Philosophical Perspectives, 30(1), 21-38.

Casati, R., \& Varzi, A. (1994). Holes and other superficialities. Cambridge: MIT Press.

Duncan, M., Miller, K., \& Norton, J. (2017). Is grounding a hyperintensional phenomenon? Analytic Philosophy, 58, 297-329.

Frisch, M. (2010). Causes, counterfactuals and non-locality. Australasian Journal of Philosophy, 88(4), 655-672.

Gilmore, C. (2013). Location and mereology. Stanford Encyclopedia of Philosophy, 1, 141-146.

Hall, N. (2002). Non-locality on the cheap? A new problem for counterfactual analyses of causation. Nous, 36(2), 276-294.

Hazlett, A. (2006). Disassembly and destruction. The Monist, 89, 418-433.

Hazlett, A. (2011). How the present depends on the future. Ratio, 24, 167-175.

Jenkins, C. S. I. (2011). Is metaphysical dependence irreflexive? The Monist, 94(2), 267-276.

Koslicki, K. (2015). The coarse-grainedness of grounding. In K. Bennett \& D. W. Zimmerman (Eds.), Oxford studies in metaphysics (Vol. 9, pp. 306-344). Oxford: Oxford University Press.

Leuenberger, S. (2013). Grounding and necessity. Inquiry, 57, 151-174.

Lewis, D., \& Lewis, S. (1970). Holes. Australasian Journal of Philosophy, 48, 206-212.

Litland, J. (2013). On some counterexamples to the transitivity of grounding. Essays in Philosophy, 14, $19-32$.

Loewer, B. (2012). Two accounts of laws and time. Philosophical Studies, 160, 115-137.

Miller, K. (2007). Immaterial beings. The Monist, 90(3), 349-371.

Raven, M. J. (2012). In defence of ground. Australasian Journal of Philosophy, 90(4), 687-701.

Rodriguez-Pereyra, G. (2015). Grounding is not a strict order. Journal of the American Philosophical Association, 1(3), 517-534. https://doi.org/10.1017/apa.2014.22.

Schaffer, J. (2009). On what grounds what. In D. Manley, D. Chalmers, \& R. Wasserman (Eds.), Metametaphysics: New essays on the foundations of ontology. Oxford: Oxford University Press.

Schaffer, J. (2012). Grounding, transitivity and contrastivity. In F. Correia \& B. Schneider (Eds.), Grounding and explanation. Cambridge: Cambridge University Press.

Schaffer, J. (2016). Grounding in the image of causation. Philosophical Studies, 173, 49-100.

Tognazzini, N. A. (2015). Grounding the luck objection. Australasian Journal of Philosophy, 93(1), $127-138$.

Trogdon, K. (2013). An Introduction to Grounding. In M. Hoeltje, B. Schnieder, \& A. Steinberg (Eds.), Varieties of dependence: Ontological dependence, grounding, supervenience, response-dependence (Basic philosophical concepts) (pp. 97-122). Munich: Philosophia Verlag.

Williamson, T. (2013). Modal logic as metaphysics. Oxford: Oxford University Press.

Wilson, J. (2014). No work for a theory of grounding. Inquiry, 57(5-6), 1-45.

Wilson, A. (2018). Metaphysical Causation. Nous, 52(4), 723-751.

Wilson, A. (forthcoming). Classifying dependencies. In D. Glick, G. Darby, \& A. Marmodoro, (Eds.), The foundation of reality: Fundamentality, space and time. Oxford: Oxford University Press.

Publisher's Note Springer Nature remains neutral with regard to jurisdictional claims in published maps and institutional affiliations. 